\title{
A Wide Passband SAW Notch Filter
}

\author{
Zhiqun Lin \\ College of Science \\ Hunan Agricultural University \\ Changsha, 410128, China \\ Jiuling Liu \\ Institute of Acoustics \\ the Chinese Academy of Sciences \\ Beijing, 100080, China \\ E-mail: lzqphy@163.com \\ Wenhui Ren \\ College of Science \\ Hunan Agricultural University \\ Changsha, 410128, China \\ Shitang $\mathrm{He}$ \\ Institute of Acoustics \\ the Chinese Academy of Sciences \\ Beijing, 100080, China
}

\begin{abstract}
Wide passband notch filter is fulfilled by combining LC-all-pass filter with one-port-SAW resonator by means of its impedance characteristics. It possesses good frequency selectivity and low insertion loss due to good frequency selectivity of resonance-frequency resonator and low insertion loss of LC filter. Higher attenuation of specified frequency can be achieved by cascade. Compared with LC notch filter, it posses sharp cutoff frequency and narrower notch width. This paper first discusses the principle of this filter, and then proceeds with theoretical simulation and experimental demonstration.
\end{abstract}

Keywords: SAW, Resonator, Notch filter

\section{Introduction}

SAW notch filter is usually applied to coding and decoding system of TV(Wu Yili,1983, pp.204-207). Wide passband notch filter is fulfilled by combining LC-all-pass filter with one-port-SAW resonator by means of its impedance characteristics(O.Ikata,1992, PP.111-115). It inherited good qualities of both SAW filter and LC-circuit and thus it possesses good frequency selectivity, large working frequency and low passband insertion loss(Gopani,1990,pp.1-5). The cascade of SAW notch filter could obtain high attenuation in particular frequency band. Compared with LC notch filter, it has remarkable frequency selectivity for its sharp-cutoff frequency characteristics. Anenna duplexer used in mobile terminals, commonly takes the ladder structure as the transmitting end filter (Hikita M,1993,pp.15-23),but this kind of structure cannot meet the request for anenna duplexer of the high demand of isolation. By coupling this notch filter with a ladder-type SAW filter, low-insertion loss in passband and high attenuation in specified frequency can be achieved(Lin Zhiqun,2006,pp.376-379). With the longitudinally coupled resonator(Moriata T,1999,pp.95-104) coupled with the SAW notch filter,its frequency response can be improved(Lin Zhiqun,2007,pp.247-249).

In this paper the theory and approaches of achieving the notch filter by combining LC-all-pass filter with one-port-SAW resonator is discussed at first, then theoretical simulation is proceeded, and ultimately experimental results are accomplished. This notch filter is characterized by small size, low insertion loss, wide passband and narrow notch frequency band.

\section{Structure and theoretical analyses of SAW notch filter}

Above-mentioned SAW notch filter is achieved by substituting capacitance in LC-all-pass network (figure 2) for 
one-port-SAW resonator (SAWR) (figure 1), and it is illustrated in figure 3.

As figure 1, the structure of one-port-SAW resonator (SAWR) shows, the middle part is interdigital. On the left and right are two metal gratings. And its impedance characteristics are illustrated in figure 4, the solid line represents the resistance, and the broken line represents the reactance. Impedance characteristics of one-port-SAW resonator (SAWR) shows that when frequency $f$ is less than the resonance frequency of the resonator $\mathrm{Fr}(\mathrm{f}<\mathrm{Fr})$, the impedance characteristics of the resonator appears capacitive; when $\mathrm{f}=\mathrm{Fr}$, the reactance of the resonator is equal to zero while the resistance tends to zero; when frequency $\mathrm{f}$ is near the anti-resonance frequency of the resonator Fa, the impedance characteristics of the resonator tends to the maximum. The needed notch filter is achieved by utilizing its impedance characteristics and combining it with LC-all-pass network.

The above notch filter has narrow stop band at the frequency of the anti-resonance frequency of the SAW resonator; meanwhile it has the same frequency response characteristics as the original LC circuit at other frequencies. The notch frequency is mainly determined by the anti-resonance frequency of the SAW resonator, which leads to good frequency selectivity. The notch frequency band could be controlled by adjusting the difference of resonance-frequency between the two resonators. With the increase of difference, the notch frequency band broadens while the attenuation in notch frequency decreases. So the difference should be adjusted in accordance with our needs.

\section{Theoretical simulations of frequency response characteristics of notch filter}

In figure $3, Z_{\mathrm{s} 1}, Z_{\mathrm{s} 2}$ represent the impedance of two one-port-SAW resonators, theoretical frequency response of one-stage SAW notch filter is:

$S_{21}=\frac{R_{D}}{1+R_{i n}\left(\frac{1}{R_{A} j \omega L_{2}}+\frac{1}{R_{A} Z_{s 2}}+R_{D} R_{F}+\frac{1}{j \omega L_{1}}\right)}$

in which

$R_{A}=1+\frac{Z_{s 1}}{j \omega L_{2}}+\frac{Z_{s 1}}{Z_{s 2}} \quad R_{B}=1+\frac{R_{0}}{j \omega L_{1}}+\frac{R_{0}}{Z_{s 2}}$

$R_{C}=1-\frac{Z_{s 1} R_{0}}{R_{A} R_{B} Z_{s 2}^{2}} \quad \quad R_{D}=\frac{R_{0}}{R_{C} R_{B} j \omega L_{1}}+\frac{R_{0}}{R_{A} R_{B} R_{C} Z_{s 2}}$

$R_{F}=\frac{Z_{s 1}}{Z_{s 2} R_{A} j \omega L_{2}}+\frac{Z_{s 1}}{R_{A} Z_{s 2}^{2}}-\frac{1}{j \omega L_{1}}-\frac{1}{Z_{s 2}}$

$\mathrm{R}_{0}$ is loaded impedance, $\mathrm{R}_{\text {in }}$ is source impedance.

In accordance with formula (1), theoretical simulation of frequency response of one-stage structure is conducted (as illustrated in figured 3). ST quartz is applied in SAW resonator. Its central frequency is $217 \mathrm{MHz}$ and its periphery inductance is $\mathrm{L} 1=145 \mathrm{nH}, \mathrm{L} 2=40 \mathrm{nH}$ respectively.

Simulation results are shown in figure 5 (power impedance and loaded impedance are both 50 ohm); figure A demonstrates the frequency response characteristics of narrow frequency domain and figure B demonstrates the frequency response characteristics of wide frequency domain $(0 \sim 900 \mathrm{MHz})$. As shown in simulation results: attenuation in notch frequency band amounts to more than $30 \mathrm{~dB}$, passband insertion loss less than $1.5 \mathrm{~dB}$ and the ripple less than $2 \mathrm{~dB}$ in the whole passband (from $0 \mathrm{~Hz}$ to $900 \mathrm{MHz}$ ). Deeper attenuation is obtained by multiple-stage structure when needed.

\section{Experimental results}

The experimental devices are fabricated on the piezoelectric crystal ST-X quartz in accordance with the two-stage of the structure shown in figure 3. The experimental characteristics curves are shown in figure 6.

As shown in the experimental result: attenuation in notch frequency f0 in two-stage structure amounts to more than $50 \mathrm{~dB}$, while at the frequency of $\mathrm{f} 0 \pm 1.5 \mathrm{MHz}$ the insertion loss is less than $1.5 \mathrm{~dB}$. Compared with LC notch filter, it has much sharper cutoff frequency characteristics. The experimental results are approximately coincident with the theoretical simulation except that the loss in high frequency of the experimental results is more than that of simulation results (about $1 \mathrm{~dB}$ ). This is somehow caused by the characteristics of inductance in high frequency.

\section{Conclusion}

Accomplishing wide passband notch filter by one-port-SAW resonator is elaborated, and theoretical simulation and experimental result are simultaneously provided in this paper. The accomplished filter inherits good qualities of both SAW resonator and LC-circuit and thus it is characterized by small size, low insertion loss, wide passband and narrow 
notch frequency band. High attenuation to particular frequency is obtained by multiple-stage structure.

\section{References}

Gopani S, \& Horine B A. (1990). 'SAW waveguide-coupled resonator notch filter'. IEEE Ultrasonics Symposium., pp. $1-5$

Hikita M, Shibagakin, \&Akagi T, et al, (1993). IEEE Ultrasonics Symposium. pp. 15-24.

Lin Zhiqun, Ren , Liu Jiuling, \&He Shitang (2007). PIEZOELECTECYRICS \& ACOUSTOOPTICS. SAW Notch Filter Coupled with Longitudinally Coupled Resonator, 29, 247-249.

Lin Zhiqun, Ren Wenhui, Liu Jiuling, \&He Shitang (2006). Applied Acoustics. SAW Notch Filter Coupled with Ladder-type Filter, 25, 376-379.

Morita T, Watanabe Y, \&Tanaka M, et al (1992). Wideband low loss double mode SAW filters. Arizona: IEEE Ultras Sym Proc Tucson. pp. 95-104.

O.Ikata, T.Miyashita, \& T.Matsuda, et al, (1992). 'DEVELOPMENT OF LOW-LOSS BAND-PASS FILTERS USING SAW RESONATORS FOR PORTABLE TELEPHONES', IEEE Ultrasonics Symposium. PP. 111-115.

Wu Yili, Liu Shenggang, \& Wang Yongde. (1983). Theory of SAW and Its Application in Electronics Technology. BeiJing: pp. 204-207.

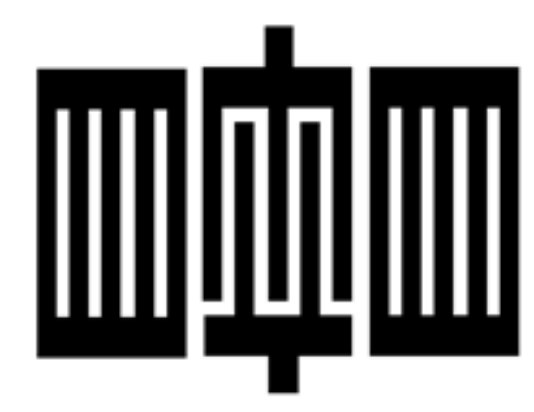

Figure 1. one-port-SAW resonator

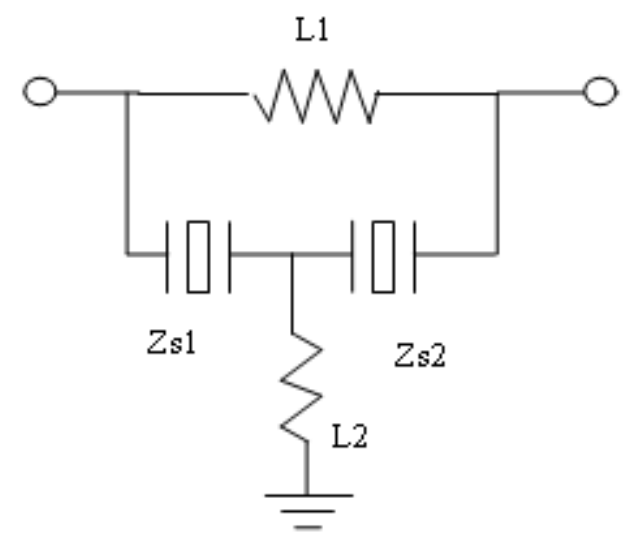

Figure 3. structure of SAW notch filter

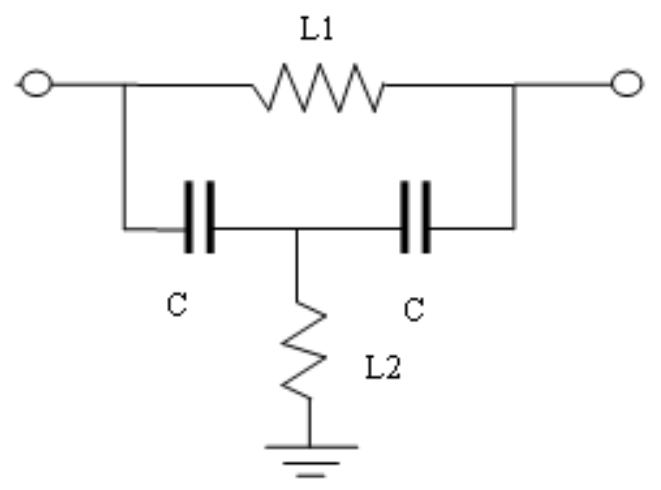

Figure 2. LC-all-pass network

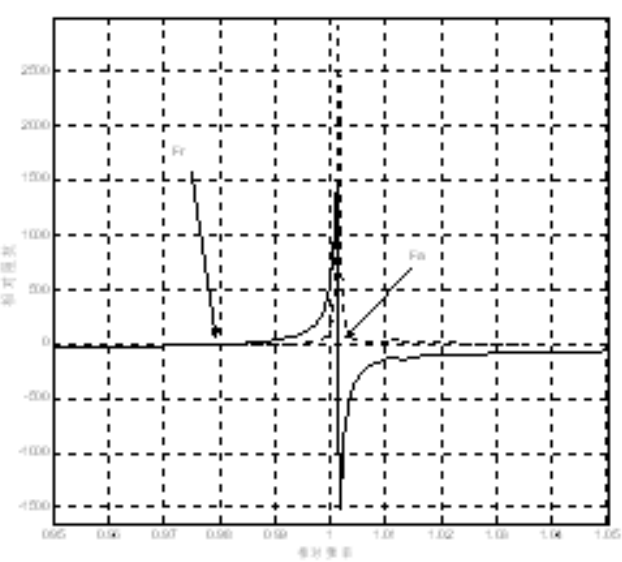

Figure 4. impedance characteristics 


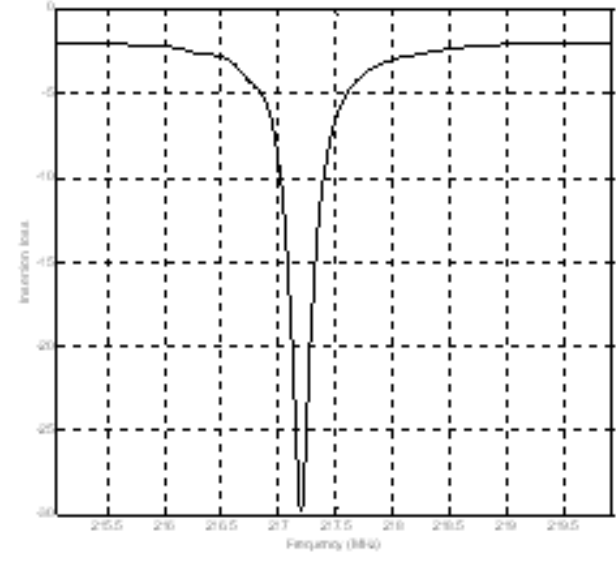

A narrow frequency domain

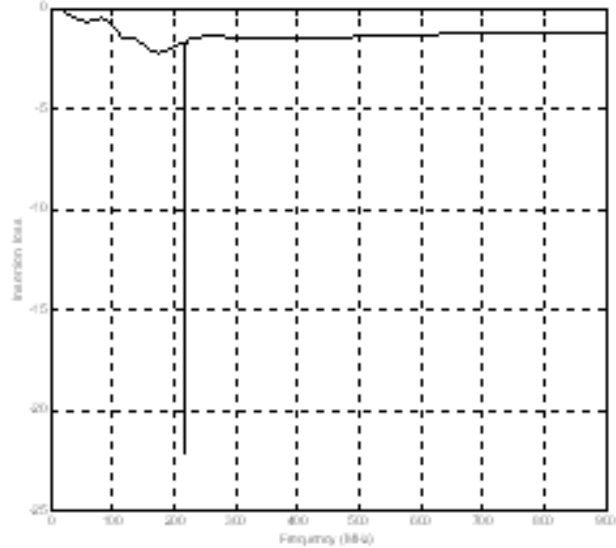

B wide frequency domain

Figure 5. simulation results of one-stage structure

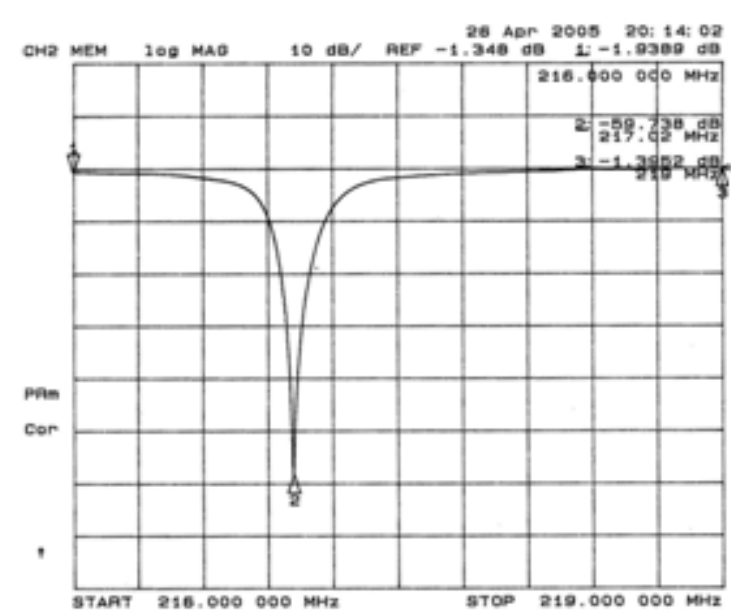

A narrow frequency domain

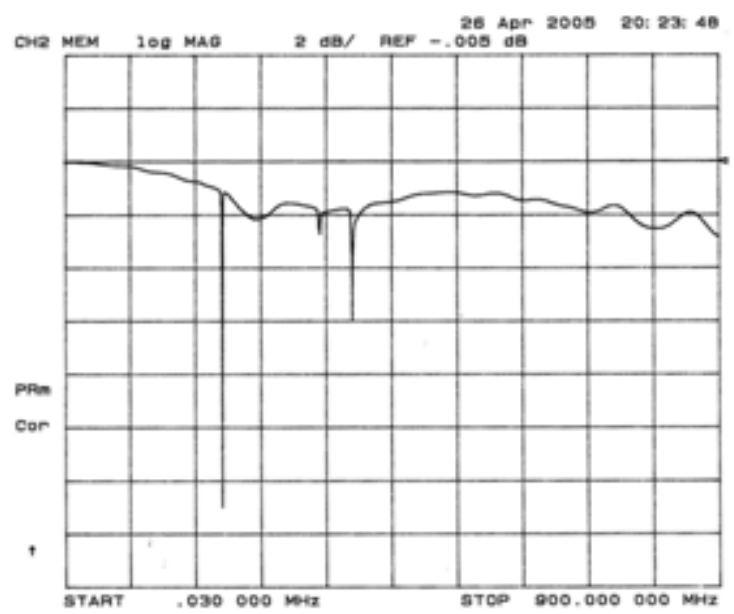

B wide frequency domain

Figure 6. experimental frequency response 